Research Article

\title{
Compact and Noncompact Solutions to Generalized Sturm-Liouville and Langevin Equation with Caputo-Hadamard Fractional Derivative
}

\author{
Ahmed Salem (D), ${ }^{1}$ Noorah Mshary, ${ }^{1,2}$ Moustafa El-Shahed, ${ }^{3}$ and Faris Alzahrani ${ }^{1}$ \\ ${ }^{1}$ Department of Mathematics, Faculty of Science, King Abdulaziz University, P.O.Box 80203, Jeddah 21589, Saudi Arabia \\ ${ }^{2}$ Department of Mathematics, Faculty of Science, Jazan University, P.O.Box 45142, Jazan 2097, Saudi Arabia \\ ${ }^{3}$ Unaizah Faculty of Arts and Sciences, Qassim University, P.O. Box 3771, Unaizah, Qassim 51431, Saudi Arabia
}

Correspondence should be addressed to Ahmed Salem; ahmedsalem74@hotmail.com

Received 3 July 2021; Revised 21 July 2021; Accepted 8 November 2021; Published 23 November 2021

Academic Editor: Shahram Rezapour

Copyright (C) 2021 Ahmed Salem et al. This is an open access article distributed under the Creative Commons Attribution License, which permits unrestricted use, distribution, and reproduction in any medium, provided the original work is properly cited.

In this work, through using the Caputo-Hadamard fractional derivative operator with three nonlocal Hadamard fractional integral boundary conditions, a new type of the fractional-order Sturm-Liouville and Langevin problem is introduced. The existence of solutions for this nonlinear boundary value problem is theoretically investigated based on the Krasnoselskii in the compact case and Darbo fixed point theorems in the noncompact case with aiding the Kuratowski's measure of noncompactness. To demonstrate the applicability and validity of the main gained findings, some numerical examples are included.

\section{Introduction}

Over the past few years, the fractional calculus (which is basically an expansion of the traditional calculus) has provided its remarkable contribution in addressing lots of physical and biological phenomena as well as describing many engineering dilemmas [1]. It has been confirmed in several literature studies that many real-world problems associated with many applied science fields can be described more convenient using the fractional-order differential equations (FoDEs) rather than that of using the ordinary differential equations (ODEs) [2]. In particular, the FoDEs played and still play a significant role in developing lots of models that outline several engineering problems and physical applications such as electromagnetics, porous media, control, and viscoelasticity [3]. For further facts on the basic principles of fractional calculus and the FoDEs, the reader may return to the references [4-7]. The fractional calculus, new interesting research field, is attracting the interest of mathematicians and researchers. With fractional operators, many real-world problems are being investigated, in particular the presenting of mathematical model for the transmission of COVID-19 and mathematical models of HIV/AIDS and drug addiction in prisons [8].

The so-called fractional-order Langevin equation (FoLE) (which was first established in [9]) is considered as a practical tool employed in describing some physical phenomena's evolution [10]. For instance, some operations related to the porous media and the reaction-diffusion can be outlined using this type of equations (see [11-13]). From these motivations, we find that exploring the existence of solutions for such equation will further enrich our understanding and knowledge about this subject. Actually, lots of results related to the solution's existence and uniqueness of the FoLE (in view of some well-known operators such as the Riemann-Liouville and Caputo operators) were extensively deduced through several research papers (see, e.g., [14-17]). For the purpose of studying such kind of problems, several strategies and schemes could be implemented such as the coincidence theory [18], the upper and lower solution method [19, 20], the fixed point theorems [21, 22], and many others. For example, some convenient findings associated with the solution's existence of the FoLE have been pointed out in [23]. Besides, this aspect together with the solution's 
uniqueness aspect of the same equation have been well explored in [24]. However, for further recent outcomes, we refer the reader to papers $[25,26]$ and references therein.

The Sturm-Liouville operator has several applications in variety fields of science such as engineering and mathematics [27]. The authors in [28] presented an approach to the fractional version of the Sturm-Liouville problem. Kiataramkul et al. [10] combined Sturm-Liouville and Langevin fractional differential equations and they called generalized Sturm-Liouville and Langevin equation (GSLLE). Furthermore, they studied the existence and uniqueness of solutions for GSLLE with antiperiodic boundary conditions.

In 1892, another fractional derivative, which is called later as the Hadamard fractional derivative, was proposed by Hadamard. This derivative is distinguished from the others by its construction that includes a logarithmic function of arbitrary exponent. Latest achievements about this derivative and its corresponding Hadamard FoDEs can be found in [29]. In [30], Jarad et al. proposed the so-called Caputo-Hadamard fractional-order derivative, which is a new modification of the Hadamard fractional derivative operator. This new version has proved its suitability in dealing with some physical interpretable initial conditions [31]. In order to get a full overview about this operator and its properties, we refer the reader to the same reference [30]. From the perspective that asserts, there are limited results in literature that addresses the boundary value problems (BVPs) in view of the Caputo-Hadamard derivative [31].

This paper attempts to present a theoretical study about the existence of solutions for the nonlinear GSLLE,

$$
{ }^{\mathrm{CH}} D^{\mu}\left(\alpha(t){ }^{\mathrm{CH}} D^{\gamma}+\beta(t)\right) x(t)=h\left(t, x(t),{ }^{\mathrm{CH}} D^{\ell} x(t)\right),
$$

subject to the following three point integral boundary conditions:

$$
x(1)=0, x(\zeta)=a_{1}{ }^{H} J^{\rho_{1}} x(\zeta),(T)=a_{2}{ }^{H} J^{\rho_{2}} x(T),
$$

where ${ }^{\mathrm{CH}} D^{\mu}$ is the Caputo-Hadamard derivative operator of order $\mu,{ }^{H} J^{\gamma}$ is the Hadamard integral operator of order $\gamma$, and $1 \leq t \leq T, 0<\ell<\gamma, 0<\gamma<1, \rho_{1}, \rho_{2}>0$, and $1<\mu<2$. The functions $\alpha:[1, T] \longrightarrow \mathbb{R}_{+}, \beta:[1, T] \longrightarrow \mathbb{R}$ and $h:[1, T] \times$ $\mathbb{R} \times \mathbb{R} \longrightarrow \mathbb{R}$ are continuous. It is remarkable to point out that when $\beta(t)=0$ for all $t \in[1, T]$, then our problem reduces to the Sturm-Liouville fractional BVP and when $\alpha(t)$ and $\beta(t)$ are real constants functions for all $t \in[1, T]$, then our problem reduces to the Langevin fractional BVP.

Here, we investigate the existence of solution in two cases: in the compact case by utilizing the well-known fixed point theorem due to Krasnoselskii. In the noncompact case, we apply the Kuratowski's measure of noncompactness technique through using the Darbo's fixed point theorems. The underlying idea is there are two measures of noncompactness commonly used; these associate numbers to sets in such a way that compact sets all get the measure 0 , and other sets get measures that are bigger according to "how far" they are removed from compactness. There are many contributors who used measure of noncompactness to investigate the existence of solution to FoDE [32-34] and references given therein.

The remaining of this paper is ordered as follows: Section 2 presents briefly some fundamental concepts related to the fractional calculus and fixed point theorems. In Section 3, some mathematical preparations and principles are established to achieve our goals through defining some essential terms and deducing some auxiliary lemmas. Section 4 employs these preparations to attain the main results of this work, which concern with exploring the existence of solution for the GSLLE. Section 5 illustrates two numerical examples followed by Section 6 that outlines the major points and concluded remarks.

\section{Preliminaries}

This part aims to present some fundamental fixed point theorems and essential preliminaries related to the fractional calculus, especially in regard to the Hadamard fractionalorder operators.

Definition 1 (see [29]). The Hadamard integral operator of the fractional-order value $\mu \in \mathbb{R}^{+}$for a function $f \in L^{p}[a, b]$ is outlined as

$$
{ }^{H} J_{a}^{\mu} f(t)=\frac{1}{\Gamma(\mu)} \int_{a}^{t}\left(\log \frac{t}{s}\right)^{\mu-1} f(s) \frac{\mathrm{d} s}{s},
$$

where $0 \leq a \leq t \leq b<\infty$.

Definition 2 (see [30]). The Caputo-Hadamard derivative operator of fractional-order $\mu$ for at least $n$-times differentiable function $f:[a, \infty) \longrightarrow \mathbb{R}$ is outlined as

$$
{ }^{\mathrm{CH}} D_{a}^{\mu} f(t)=\frac{1}{\Gamma(n-\mu)} \int_{a}^{t}\left(\log \frac{t}{s}\right)^{n-\mu-1} \delta^{n} f(s) \frac{\mathrm{d} s}{s},
$$

where $\quad n-1<\mu<n, \quad n=[\mu]+1, \quad \delta=t \mathrm{~d} / \mathrm{d} t, \quad$ and $\log (\cdot)=\log _{e}(\cdot)$.

Lemma 1 (see [30]). Suppose $f \in C[a, b]$ and $\mu>0$ such that $n=[\mu]+1$. Then,

$$
{ }^{\mathrm{CH}} D_{a}^{\mu}\left({ }^{H} J_{a}^{\mu} f\right)(t)=f(t) .
$$

Lemma 2 (see [30]). Let $f \in A C_{\delta}^{n}[a, b]$ or $C_{\delta}^{n}[a, b]$ and $\mu>0$ such that $n=[\mu]+1$. Then,

$$
\left({ }^{H} J_{a}^{\mu} \cdot{ }^{\mathrm{CH}} D_{a}^{\mu} f\right)(t)=f(t)-\sum_{k=0}^{n-1} c_{k}\left(\frac{\log t}{a}\right)^{k},
$$

where $C_{\delta}^{n}[a, b]=\left\{f:[a, b] \longrightarrow \mathbb{C}: \delta^{n-1} f(t) \in C[a, b]\right\}$.

Lemma 3 (see $[30,29]$ ). Let $\mu, \nu>0$, then we have

(i) ${ }^{H} J_{a}^{\mu} H J_{a}^{\nu} f(t)={ }^{H} J_{a}^{\mu+v} f(t)$,

(ii) ${ }^{H} J_{a}^{\mu} \log ^{\nu-1}(t / a)=\Gamma(\nu) / \Gamma(\nu+\mu) \log ^{\mu+\nu-1}(t / a)$,

(iii) ${ }^{\mathrm{CH}} D_{a}^{\mu} H J_{a}^{\mu} f(t)={ }^{H} J_{a}^{\mu-v} f(t), \mu>v$,

(iv) ${ }^{\mathrm{CH}} D_{a}^{\mu} \log ^{\nu-1}(t / a)=\Gamma(\nu) / \Gamma(\nu-v) \log ^{\nu-\mu-1} \quad(t / a), v$ $\neq 1,2, \ldots, n-1, n=[\mu]+1$, 
(v) ${ }^{\mathrm{CH}} D_{a}^{\mu} \log ^{\nu-1} \quad(t / a)=0, v=1,2, \ldots, n-1, n=[\mu]$ +1 ,

where $f \in L^{p}(a, b)$, and $1 \leq p<\infty$.

For simplicity, we will consider $a=1$ in all aforementioned preliminaries, and consequently ${ }^{H} J_{a}^{\mu}$ and ${ }^{H} D_{a}^{\mu}$ will be denoted by $J^{\mu}$ and $D^{\mu}$, respectively. However, some essential fixed point theorems are reported below for completeness.

Definition 3 (see [3]). Suppose that $\mathscr{B}$ is a Banach space and $\Upsilon_{\mathscr{B}}$ is the collection of subsets of $\mathscr{B}$. The Kuratowski's measure of noncompactness $\sigma: \Upsilon_{\mathscr{B}} \longrightarrow[0, \infty]$ is a map outlined as

$$
\sigma(X)=\inf \left\{\kappa>0: X \subseteq \bigcup_{i=1}^{n} X_{i} \text { and } \operatorname{diam}\left(X_{i}\right) \leq \kappa\right\},
$$

where

$$
\operatorname{diam}\left(X_{i}\right)=\sup \left\{\left\|x_{1}-x_{2}\right\|: x_{1}, x_{2} \in X_{i}\right\}
$$

Lemma 4 (see [3]). Suppose that $\mathscr{B}$ is a Banach space, $X, X_{1}$, and $X_{2}$ are bounded sets of $\mathscr{B}$, and $\sigma$ is the Kuratowski's measure of noncompactness defined above. Then, we have the following assertions:

(i) $X$ is compact $\Longleftrightarrow \sigma(X)=0$.

(ii) $\sigma\left(X_{1}+X_{2}\right) \leq \sigma\left(X_{1}\right)+\sigma\left(X_{2}\right)$, where $X_{1}+X_{2}=\left\{x_{1}\right.$ $\left.+x_{2}: x_{1} \in X_{1}, x_{2} \in X_{2}\right\}$.

(iii) $\sigma(\varrho X)=|\varrho| \sigma(X)$, where $\varrho X=\{\varrho x: x \in X, \varrho \in \mathbb{R}\}$.

(iv) $\sigma\left(X+x_{0}\right)=\sigma(X)$, for any $x_{0} \in \mathscr{B}$.

(v) If the map $F: C([a, b], \mathbb{R}) \longrightarrow \mathscr{B}$ is bounded and equicontinuous, it follows

$$
\sigma\left(\int_{a}^{t} F(s) \mathrm{d} s\right) \leq \int_{a}^{t} \sigma(F(s)) \mathrm{d} s .
$$

Lemma 5 (Ascoli-Arzela theorem [3]). The family $\mathscr{F}=\{f(t)\}$ in $C([0, T], \mathscr{B})$ has a uniformly-convergent subsequence $\left\{f_{n}(t)\right\}_{n=1}^{\infty}$, if it is uniformly bounded and equicontinuous maps on $[0, T]$, and $\left\{f\left(t_{0}\right)\right\}$ is relatively compact in the Banach space $\mathscr{B}$, for any $t_{0} \in[0, T]$.

Theorem 1 (Krasnoselskii's fixed point theorem $[3,26])$. Suppose that $\mathscr{C}_{\varepsilon}$ is a closed convex bounded set of a Banach space $\mathscr{B}$, and $S_{1}, S_{2}: \mathscr{B} \longrightarrow \mathscr{B}$ are two operators satisfying

(1) $S_{1} x_{1}+S_{2} x_{2} \in \mathscr{C}_{\varepsilon}$, for each $x_{1}, x_{2} \in \mathscr{C}_{\varepsilon}$.

(2) $S_{2}$ is a contraction mapping.

(3) $S_{1}$ is continuous and $S_{1} \mathscr{C}_{\varepsilon}$ is a compact subset of $\mathscr{B}$.

Then, $S=S_{1} x+S_{2} x$ has a fixed point.

Theorem 2 (Darbo's fixed point theorem [3]). Suppose that $\mathscr{C}_{\varepsilon}$ is a closed convex bounded set of a Banach space $\mathscr{B}$, and $S: \mathscr{C}_{\varepsilon} \longrightarrow \mathscr{C}_{\varepsilon}$ is a continuous mapping so that

$$
\sigma(S(W)) \leq k \sigma(W)
$$

for all closed subsets $W \in \mathscr{C}_{\mathcal{E}}$, where $0 \leq k<1$. Then, $S$ has a fixed point in $\mathscr{C}_{\varepsilon}$.

\section{Auxiliary Results}

This section presents some auxiliary results that will provide a basis for introducing some results associated with the existence of the solutions for the GSLLE.

Lemma 6. The linear BVP given in the following

$$
{ }^{\mathrm{CH}} D^{\mu}\left(\alpha(t){ }^{\mathrm{CH}} D^{\gamma}+\beta(t)\right) x(t)=g(t),
$$

subjects to the conditions in (2) which is equivalent to the following fractional-order integral equation:

$$
\begin{aligned}
x(t)= & { }^{H} J^{\gamma}\left[\frac{{ }^{H} J^{\mu} g(t)-\beta(t) x(t)}{\alpha(t)}\right]+\frac{\chi(t, T)}{\chi(\zeta, T)}\left(a_{1}{ }^{H} J^{\rho_{1}} x(\zeta)-{ }^{H} J^{\gamma}\left[\frac{{ }^{H} J^{\mu} g(\zeta)-\beta(\zeta) x(\zeta)}{\alpha(\zeta)}\right]\right) \\
& +\frac{\chi(t, \zeta)}{\chi(T, \zeta)}\left(a_{2}{ }^{H} J^{\rho_{2}} x(T)-{ }^{H} J^{\gamma}\left[\frac{{ }^{H} J^{\mu} g(T)-\beta(T) x(T)}{\alpha(T)}\right]\right)
\end{aligned}
$$

where $g:[1, T] \longrightarrow \mathbb{R}$ is a continuous function and the other concepts are defined as in the Introduction section, $\chi(\zeta, T) \neq 0$, 


$$
\begin{aligned}
& \chi(t, s)=b_{1}(t) b_{2}(s)-b_{2}(t) b_{1}(s)=\left|\begin{array}{ll}
b_{1}(t) & b_{2}(t) \\
b_{1}(s) & b_{2}(s)
\end{array}\right|, \\
& b_{i}(t)=\frac{1}{\Gamma(\gamma)} \int_{1}^{t}\left(\log \frac{t}{s}\right)^{\gamma-1} \log ^{i-1} s \frac{\mathrm{d} s}{s \alpha(s)}, \quad i \in\{1,2\} .
\end{aligned}
$$

Proof. Applying the Hadamard fractional-order integral ${ }^{H} J^{\mu}$ to both sides of (11) yields

$$
\alpha(t){ }^{\mathrm{CH}} D^{\gamma} x(t)+\beta(t) x(t)-\left(c_{0}+c_{1} \log t\right)={ }^{H} J^{\mu} g h(t) .
$$

That is,

$$
{ }^{\mathrm{CH}} D^{\gamma} x(t)=\frac{{ }^{H} J^{\mu} g(t)-\beta(t) x(t)+c_{0}+c_{1} \log t}{\alpha(t)} .
$$

Again, taking ${ }^{H} J^{\gamma}$ to both sides of the equation above yields

$$
x(t)={ }^{H} J^{\gamma}\left[\frac{{ }^{H} J^{\mu} g(t)-\beta(t) x(t)+c_{0}+c_{1} \log t}{\alpha(t)}\right]+c_{2} .
$$

Now, using the first condition $(x(1)=0$ given in (2)) yields $c_{2}=0$, and then we have

$$
x(t)={ }^{H} J^{\gamma}\left[\frac{{ }^{H} J^{\mu} g(t)-\beta(t) x(t)}{\alpha(t)}\right]+b_{1}(t) c_{0}+b_{2}(t) c_{1} .
$$

On the other hand, using the other two boundary conditions given in (2) leads us to the following two assertions:

$$
\begin{aligned}
& a_{1}{ }^{H} J^{\rho_{1}} x(\zeta)={ }^{H} J^{\gamma}\left[\frac{{ }^{H} J^{\mu} g(\zeta)-\beta(\zeta) x(\zeta)}{\alpha(\zeta)}\right]+b_{1}(\zeta) c_{0}+b_{2}(\zeta) c_{1}, \\
& a_{2}{ }^{H} J^{\rho_{2}} x(T)={ }^{H} J^{\gamma}\left[\frac{{ }^{H} J^{\mu} g(T)-\beta(T) x(T)}{\alpha(T)}\right]+b_{1}(T) c_{0}+b_{2}(T) c_{1}
\end{aligned}
$$

Solving these two equations gives, respectively, the following two expressions of $c_{0}$ and $c_{1}$ :

$$
\begin{aligned}
c_{0}= & \frac{1}{\chi(\zeta, T)}\left(a_{1} b_{2}(T)^{H} J^{\rho_{1}} x(\zeta)-a_{2} b_{2}(\zeta)^{H} J^{\rho_{2}} x(T)-b_{2}(T)^{H} J^{\gamma}\left[\frac{{ }^{H} J^{\mu} g(\zeta)-\beta(\zeta) x(\zeta)}{\alpha(\zeta)}\right]\right. \\
& \left.+b_{2}(\zeta)^{H} J^{\gamma}\left[\frac{{ }^{H} J^{\mu} g(T)-\beta(T) x(T)}{\alpha(T)}\right]\right), \\
c_{1}= & \frac{-1}{\chi(\zeta, T)}\left(a_{1} b_{1}(T)^{H} J^{\rho_{1}} x(\zeta)-a_{2} b_{1}(\zeta)^{H} J^{\rho_{2}} x(T)-b_{1}(T)^{H} J^{\gamma}\left[\frac{{ }^{H} J^{\mu} g(\zeta)-\beta(\zeta) x(\zeta)}{\alpha(\zeta)}\right]\right. \\
& \left.+b_{1}(\zeta)^{H} J^{\gamma}\left[\frac{{ }^{H} J^{\mu} g(T)-\beta(T) x(T)}{\alpha(T)}\right]\right) .
\end{aligned}
$$

Finally, substituting $c_{0}$ and $c_{1}$ in (18) yields the desired result.

Lemma 7. Let $t \in[1, T]$ and $s \in(1, T]$. Then, for the function $\chi(t, s)$ defined in (13), we can deduce the following assertion:

$$
\chi_{0}(s)=\max _{t \in[1, T]}|\chi(t, s)|=\left(\frac{\alpha_{2}}{b_{1}(s)}\right)^{\gamma}\left(\frac{b_{2}(s)}{\alpha_{1}}\right)^{1+\gamma} \frac{\gamma^{\gamma}}{\Gamma(\gamma+2)},
$$

where $\alpha_{1}=\min _{t \in[1, T]} \alpha(t)$ and $\alpha_{2}=\max _{t \in[1, T]} \alpha(t)$.

Proof. Since the function $\alpha:[1, T] \longrightarrow \mathbb{R}_{+}$is continuous, then it attains its minimum and maximum which leads to

$$
\frac{1}{\alpha_{2}}{ }^{H} J^{\gamma} \log ^{i-1} t \leq b_{i}(t) \leq \frac{1}{\alpha_{1}}{ }^{\gamma} J^{\gamma} \log ^{i-1} t, \quad i=1,2,
$$

where $b_{i}, i=1,2$ are defined in (14). In view of Lemma 3, we get 
$\frac{1}{\alpha_{2} \Gamma(\gamma+i)} \log ^{\gamma+i-1} t \leq b_{i}(t) \leq \frac{1}{\alpha_{1} \Gamma(\gamma+i)} \log ^{\gamma+i-1} t, \quad i=1,2$.

Whence,

$$
\chi(t, s) \leq \frac{b_{1}(s) f(t)}{\alpha_{2} \Gamma(\gamma+2)}
$$

where

$$
f(t)=\frac{\alpha_{2} b_{2}(s)(1+\gamma)}{\alpha_{1} b_{1}(s)} \log ^{\gamma} t-\log ^{\gamma+1} t .
$$

Differentiation gives

$$
f^{\prime}(t)=\frac{\gamma+1}{t} \log ^{\gamma-1} t\left[\frac{\alpha_{2} b_{2}(s) \gamma}{\alpha_{1} b_{1}(s)}-\log t\right],
$$

which means that the function $f$ is increasing on $\left(1, t_{0}\right)$ and decreasing on $\left(t_{0}, T\right)$ where $t_{0}=e^{\alpha_{2} b_{2}(s) \gamma / \alpha_{1} b_{1}(s)}$. Thus, $f(t) \leq f\left(t_{0}\right)$ for all $t \in[1, T]$ which implies the desired result.

In this work, the Banach space of all continuous realvalued functions $\mathscr{B}=C([1, T], \mathbb{R})$ will be considered in which it is equipped with the usual maximum norm:

$$
\|x\|=\max _{1 \leq t \leq T}|x(t)|, \quad x \in \mathscr{B} .
$$

Define the space

$$
\mathscr{Y}=\left\{x(t): x(t) \in \mathscr{B},{ }^{\mathrm{CH}} D^{\ell} x(t) \in \mathscr{B}\right\}, \quad t \in[1, T],
$$

as a subset of $\mathscr{B}$, then one can easily check that $\mathscr{Y}$ represents a Banach subspace equipped with the norm:

$$
\begin{aligned}
\|x\|_{y} & =\max \left\{\|x\|,\left\|{ }^{\mathrm{CH}} D^{\ell} x\right\|\right\} \\
& =\max \left\{\max _{1 \leq t \leq T}|x(t)|, \max _{1 \leq t \leq T}\left|{ }^{C H} D^{\ell}(x(t))\right|\right\} .
\end{aligned}
$$

In light of Lemma 6, the B.V.P. given in (1) and (2) can be converted into a fixed point problem. This means that the function $x(t)$ must hold the two assertions: $S x(t)=x(t)$ and ${ }^{\mathrm{CH}} D^{\ell} S x(t)={ }^{\mathrm{CH}} D^{\ell} x(t)$ for all $t \in[1, T]$, where $S: \mathscr{B} \longrightarrow \mathscr{B}$ and ${ }^{\mathrm{CH}} D^{\ell} S: \mathscr{B} \longrightarrow \mathscr{B}$ are the two operators defined on $\mathscr{B}$. For achieving this objective, we define the operator $S$ as follows:

$$
\begin{aligned}
S x(t) & =S_{1} x(t)+S_{2} x(t), \\
{ }^{\mathrm{CH}} D^{\ell} S x(t) & ={ }^{\mathrm{CH}} D^{\ell} S_{1} x(t)+{ }^{\mathrm{CH}} D^{\ell} S_{2} x(t),
\end{aligned}
$$

where

$$
\begin{aligned}
& S_{1} x(t)={ }^{H} J^{\gamma}\left[\frac{{ }^{H} J^{\mu} h\left(t, x(t),{ }^{\mathrm{CH}} D^{\ell} x(t)\right)}{\alpha(t)}\right]-\frac{\chi(t, T)}{\chi(\zeta, T)} J^{\gamma}\left[\frac{{ }^{H} J^{\mu} h\left(\zeta, x(\zeta),{ }^{\mathrm{CH}} D^{\ell} x(\zeta)\right)}{\alpha(\zeta)}\right] \\
& -\frac{\chi(t, \zeta)}{\chi(T, \zeta)}{ }^{\gamma} J^{\gamma}\left[\frac{{ }^{H} J^{\mu} h\left(T, x(T),{ }^{C H} D^{\ell} x(T)\right)}{\alpha(T)}\right] \text {, } \\
& S_{2} x(t)=-{ }^{H} J^{\gamma}\left[\frac{\beta(t) x(t)}{\alpha(t)}\right]+\frac{\chi(t, T)}{\chi(\zeta, T)}\left(a_{1}{ }^{H} J^{\rho_{1}} x(\zeta)+{ }^{H} J^{\gamma}\left[\frac{\beta(\zeta) x(\zeta)}{\alpha(\zeta)}\right]\right) \\
& +\frac{\chi(t, \zeta)}{\chi(T, \zeta)}\left(a_{2}{ }^{H} J^{\rho_{2}} x(T)+{ }^{H} J^{\gamma}\left[\frac{\beta(T) x(T)}{\alpha(T)}\right]\right) \\
& { }^{\mathrm{CH}} D^{\ell} S_{1} x(t)={ }^{H} J^{\gamma-\ell}\left[\frac{{ }^{H} J^{\mu} h\left(, x(t),{ }^{\mathrm{CH}} D^{\ell} x(t)\right)}{\alpha(t)}\right]-\frac{{ }^{\mathrm{CH}} D^{\ell} \chi(t, T)}{\chi(\zeta, T)} H J^{\gamma}\left[\frac{{ }^{H} J^{\mu} h\left(\zeta, x(\zeta),{ }^{\mathrm{CH}} D^{\ell} x(\zeta)\right)}{\alpha(\zeta)}\right] \\
& -\frac{{ }^{\mathrm{CH}} D^{\ell} \chi(t, \zeta)}{\chi(T, \zeta)}{ }^{\gamma} J^{\gamma}\left[\frac{{ }^{H} J^{\mu} h\left(T, x(T),{ }^{\mathrm{CH}} D^{\ell} x(T)\right)}{\alpha(T)}\right] \text {, } \\
& { }^{\mathrm{CH}} D^{\ell} S_{2} x(t)=-{ }^{H} J^{\gamma-\ell}\left[\frac{\beta(t) x(t)}{\alpha(t)}\right]+\frac{{ }^{\mathrm{CH}} D^{\ell} \chi(t, T)}{\chi(\zeta, T)}\left(a_{1}{ }^{H} J^{\rho_{1}} x(\zeta)+{ }^{H} J^{\gamma}\left[\frac{\beta(\zeta) x(\zeta)}{\alpha(\zeta)}\right]\right) \\
& +\frac{{ }^{\mathrm{CH}} D^{\ell} \chi(t, \zeta)}{\chi(T, \zeta)}\left(a_{2}{ }^{H} J^{\rho_{2}} x(T)+{ }^{H} J^{\gamma}\left[\frac{\beta(T) x(T)}{\alpha(T)}\right]\right) \text {. }
\end{aligned}
$$


In a similar manner in Lemma 7 , we can deduce as follows.
Lemma 8. Let $t \in[1, T]$ and $s \in(1, T]$. Then, for the function $\chi(t, s)$ defined in (13), we can deduce the following assertion:

$$
\chi_{\ell}(s)=\max _{t \in[1, T]}\left|{ }^{\mathrm{CH}} D^{\ell} \chi(t, s)\right|=\left(\frac{\alpha_{2}}{b_{1}(s)}\right)^{\gamma-\ell}\left(\frac{b_{2}(s)}{\alpha_{1}}\right)^{1+\gamma-\ell} \frac{(\gamma-\ell)^{\gamma-\ell}}{\Gamma(\gamma-\ell+2)}
$$

where $\alpha_{1}=\min _{t \in[1, T]} \alpha(t)$ and $\alpha_{2}=\max _{t \in[1, T]} \alpha(t)$.

Proof. Let $\varepsilon>0, t_{2}>t_{1}$ and $t_{2}-t_{1}<\delta$ where

Lemma 9. Let $\chi(t, \tau)$ be defined as in (13), $t_{1}, t_{2} \in[1, T]$, and $1<\tau<T$. Then, for all $\varepsilon>0$, there exists $\delta=\delta(\varepsilon)>0$ such that $\left|\chi\left(t_{2}, \tau\right)-\chi\left(t_{1}, \tau\right)\right|<\varepsilon$ whenever $\left|t_{2}-t_{1}\right|<\delta$.

$$
\delta=\min \left\{\exp \left(\frac{\alpha_{1} \varepsilon \Gamma(1+\gamma)}{b_{2}(\tau)}\right)^{1 / \gamma}-1, \exp \left(\frac{\alpha_{1} \varepsilon \Gamma(1+\gamma)}{b_{1}(\tau) \log T}\right)^{1 / \gamma}-1\right\}
$$

Then, from (14) and the fact that $0<\gamma<1$, we have

$$
\begin{aligned}
\left|b_{1}\left(t_{2}\right)-b_{1}\left(t_{1}\right)\right| & =\frac{1}{\Gamma(\gamma)}\left|\int_{1}^{t_{2}}\left(\log \frac{t_{2}}{s}\right)^{\gamma-1} \frac{\mathrm{d} s}{s \alpha(s)}-\int_{1}^{t_{1}}\left(\log \frac{t_{1}}{s}\right)^{\gamma-1} \frac{\mathrm{d} s}{s \alpha(s)}\right| \\
& \leq \frac{1}{\alpha_{1} \Gamma(\gamma)} \int_{1}^{t_{1}}\left[\left(\log \frac{t_{1}}{s}\right)^{\gamma-1}-\left(\log \frac{t_{2}}{s}\right)^{\gamma-1}\right] \frac{\mathrm{d} s}{s}+\int_{t_{1}}^{t_{2}}\left(\log \frac{t_{2}}{s}\right)^{\gamma-1} \frac{\mathrm{d} s}{s} \\
& =\frac{1}{\alpha_{1} \Gamma(\gamma+1)}\left(2\left(\log \frac{t_{2}}{t_{1}}\right)^{\gamma}+\log ^{\gamma} t_{1}-\log ^{\gamma} t_{2}\right) \\
& \leq \frac{2}{\alpha_{1} \Gamma(\gamma+1)}\left(\log \frac{t_{2}}{t_{1}}\right)^{\gamma} .
\end{aligned}
$$

Since $t_{2}-t_{1}<\delta$, then $t_{2} / t_{1}<1+\delta / t_{1}<1+\delta$. Hence,

$$
\left|b_{2}\left(t_{2}\right)-b_{2}\left(t_{1}\right)\right| \leq \frac{2 \log T}{\alpha_{1} \Gamma(\gamma+1)} \log ^{\gamma}(1+\delta)<\frac{\varepsilon}{b_{1}(\tau)} .
$$

$\left|b_{1}\left(t_{2}\right)-b_{1}\left(t_{1}\right)\right| \leq \frac{2}{\alpha_{1} \Gamma(\gamma+1)} \log ^{\gamma}(1+\delta)<\frac{\varepsilon}{b_{2}(\tau)}$.

Therefore,

Similarly, we obtain

$$
\left|\chi\left(t_{2}, \tau\right)-\chi\left(t_{1}, \tau\right)\right|=b_{2}(\tau)\left|b_{1}\left(t_{2}\right)-b_{1}\left(t_{1}\right)\right|+b_{1}(\tau)\left|b_{2}\left(t_{2}\right)-b_{2}\left(t_{1}\right)\right|<\varepsilon
$$

which ends the proof.

The previous discussion together with the aforesaid proposed results represent a preparation for the next section that presents the main outcomes of the problem under consideration.

\section{The Existence of Solution for the GSLLE}

This part intends to introduce some theoretical outcomes associated with the existence of solution for the GSLLE given in (1) and (2). From now on, we will utilize the following appropriate conditions: 
$\mathbf{H}_{1}$ : the function $h:[1, T] \times \mathbb{R} \times \mathbb{R} \longrightarrow \mathbb{R} \quad$ is continuous.

$\mathbf{H}_{2}$ : there exists a positive function $Q:[1, T] \longrightarrow \mathbb{R}^{+}$ such that

$$
\left|h\left(t, x(t), D^{\ell} x(t)\right)\right| \leq Q(t), \quad \forall t \in[1, T] .
$$

$\mathbf{H}_{3}$ : there exists a constant $L>0$ such that

$$
\left\|h\left(t, x_{1}, y_{1}\right)-h\left(t, x_{2}, y_{2}\right)\right\| \leq L \max \left\{\left|x_{1}-x_{2}\right|,\left|y_{1}-y_{2}\right|\right\},
$$

for all $t \in[1, T]$ and $x_{1}, x_{2}, y_{1}, y_{2} \in \mathscr{B}$.

For convenience, we define

$$
\begin{aligned}
\lambda_{i}(t) & =\frac{\left|a_{i}\right| \log ^{\rho_{i}} t}{\Gamma\left(\rho_{i}+1\right)}, \quad i=1,2, \\
\psi_{\ell}(\gamma) & =\frac{\log ^{\gamma T}}{\alpha_{1} \Gamma(\gamma-\ell+1)}+\frac{\chi_{\ell}(T) \log ^{\gamma} \zeta+\chi_{\ell}(\zeta) \log ^{\gamma} T}{\alpha_{1}|\chi(\zeta, T)| \Gamma(\gamma+1)}, \\
\Psi_{\ell} & =\beta^{*} \psi_{\ell}(\gamma)+\frac{\chi_{\ell}(T) \lambda_{1}(\zeta)+\chi_{\ell}(\zeta) \lambda_{2}(T)}{|\chi(\zeta, T)|},
\end{aligned}
$$

where $\beta^{*}=\max _{t \in[1, T]}|\beta(t)|$.
4.1. Compactness Case. In this subsection, we assume the compactness of solution and investigate the existence of it through employing the Krasnoselskii's fixed point theorem to deduce the first result about the existence of solution of the GSLLE.

Theorem 3. Suppose $\mathbf{H}_{1}$ and $\mathbf{H}_{2}$ hold. If

$$
\max \left\{\Psi_{0}, \Psi_{\ell}\right\}<1 \text {, }
$$

where $\Psi_{\ell}$ is defined as in (45), then there is at least one solution to the B.V.P (1) and (2) in $[1, T]$.

Proof. For the purpose of proving this theorem, the closed ball $\mathscr{C}_{\epsilon}$ is defined in the following manner:

$$
\mathscr{C}_{\varepsilon}=\left\{x(t) \in \mathcal{Y}: D^{\ell} x(t) \in \mathcal{Y},|x|_{y} \leq \varepsilon\right\},
$$

where $\varepsilon$ is the radius and assumed so that

$$
\varepsilon \geq \frac{|Q| \max \left\{\psi_{0}(\gamma+\mu), \psi_{\ell}(\gamma+\mu)\right\}}{-\max \left\{\Psi_{0}, \Psi_{\ell}\right\}},
$$

where $\psi_{\ell}(\cdot)$ is defined as in (44).

One can confirm that $\mathscr{C}_{\varepsilon}$ is a closed convex bounded set. Actually, this paves the way to implement the Krasnoselskii's fixed point theorem. To achieve this goal, we intend to prove that $S_{1} x_{1}+S_{2} x_{2} \in \mathscr{C}_{\varepsilon}, \forall x_{1}(t), x_{2}(t) \in \mathscr{C}_{\varepsilon}$. So, to perform this task, we start with the following assertion:

$$
\begin{aligned}
& \left|S_{1} x_{1}(t)\right| \leq \frac{1}{|\alpha(t)|}\left[{ }^{H} J^{\gamma+\mu}\left|h\left(t, x_{1}(t),{ }^{\mathrm{CH}} D^{\ell} x_{1}(t)\right)\right|+\frac{|\chi(t, T)|_{H}}{|\chi(\zeta, T)|} J^{\gamma+\mu}\left|h\left(\zeta, x_{1}(\zeta),{ }^{\mathrm{CH}} D^{\ell} x_{1}(\zeta)\right)\right|\right. \\
& \left.+\frac{|\chi(t, \zeta)|_{H}}{|\chi(T, \zeta)|} J^{\gamma+\mu}\left|h\left(T, x_{1}(T),{ }^{\mathrm{CH}} D^{\ell} x_{1}(T)\right)\right|\right] \\
& \left.\leq \frac{|Q|}{\alpha_{1} \Gamma(\gamma+\mu)}\left[\int_{1}^{t}\left(\log \frac{t}{s}\right)^{\gamma+\mu-1} \frac{\mathrm{d} s}{s}+\frac{\chi_{0}(T)}{|\chi(\zeta, T)|} \int_{1}^{\zeta}\left(\log \frac{\zeta}{s}\right)^{\gamma+\mu-1} \frac{\mathrm{d} s}{s}+\frac{\chi_{0}(\zeta)}{|\chi(T, \zeta)|} \int_{1}^{T}\left(\log \frac{T}{s}\right)^{\gamma+\mu-1} \frac{\mathrm{d} s}{s}\right)\right] \\
& =\frac{|Q|}{\alpha_{1} \Gamma(\gamma+\mu+1)}\left[\log ^{\gamma+\mu} t+\frac{\chi_{0}(T) \log ^{\gamma+\mu} \zeta+\chi_{0}(\zeta) \log ^{\gamma+\mu} T}{|\chi(\zeta, T)|}\right] \text {. }
\end{aligned}
$$

It is known that $\log t$ is positive and increasing on $[1, T]$ which implies that $\log ^{\gamma+\mu} t \leq \log ^{\gamma+\mu} T$. Consequently, we have

$$
\left|S_{1} x_{1}\right| \leq\|Q\| \psi_{0}(\gamma+\mu)
$$




$$
\begin{aligned}
\left|S_{2} x_{2}\right| \leq & { }^{H} J^{\gamma}\left(\frac{|\beta(t)|\left|x_{2}(t)\right|}{|\alpha(t)|}\right)+\frac{|\chi(t, T)|}{|\chi(\zeta, T)|}\left(\left|a_{1}\right| J^{\rho_{1}}\left|x_{2}(\zeta)\right|+{ }^{H} J^{\gamma}\left(\frac{|\beta(\zeta)|\left|x_{2}(\zeta)\right|}{|\alpha(\zeta)|}\right)\right) \\
& +\frac{|\chi(t, \zeta)|}{|\chi(T, \zeta)|}\left(\left|a_{2}\right|^{H} J^{\rho_{2}}\left|x_{2}(T)\right|+{ }^{H} J^{\gamma}\left(\frac{|\beta(T)|\left|x_{2}(T)\right|}{|\alpha(T)|}\right)\right) \\
& \leq\|x\|\left[\frac{\beta^{*} \log ^{\gamma} T}{\alpha_{1} \Gamma(\gamma+1)}+\frac{\chi(T)}{|\chi(\zeta, T)|}\left(\frac{\left|a_{1}\right| \log ^{\rho_{1}} \zeta}{\Gamma\left(\rho_{1}+1\right)}+\frac{\beta^{*} \log ^{\gamma} \zeta}{\alpha_{1} \Gamma(\gamma+1)}\right)+\frac{\chi(\zeta)}{|\chi(T, \zeta)|}\left(\frac{\left|a_{2}\right| \log ^{\rho_{2}} T}{\Gamma\left(\rho_{2}+1\right)}+\frac{\beta^{*} \log ^{\gamma} T}{\alpha_{1} \Gamma(\gamma+1)}\right)\right] \\
= & \|x\|\left[\beta^{*} \psi(\gamma)+\frac{\chi(T) \lambda_{1}(\zeta)+\chi(\zeta) \lambda_{2}(T)}{|\chi(\zeta, T)|}\right] \leq \varepsilon \Psi_{0} .
\end{aligned}
$$

Now, from (50) and (51), we obtain

$$
\left|S_{1} x_{1}+S_{2} x_{2}\right| \leq\|Q\| \psi_{0}(\gamma+\mu)+\varepsilon \Psi_{0} .
$$

Similarly, we obtain

$$
\begin{aligned}
& \left|{ }^{\mathrm{CH}} D^{\ell} S_{1} x_{1}\right| \leq\|Q\| \psi_{\ell}(\gamma+\mu), \\
& \left|{ }^{\mathrm{CH}} D^{\ell} S_{2} x_{2}\right| \leq \varepsilon \Psi_{\ell} .
\end{aligned}
$$

Accordingly, the two inequalities above imply

$$
\left|{ }^{\mathrm{CH}} D^{\ell} S_{1} x_{1}+{ }^{\mathrm{CH}} D^{\ell} S_{2} x_{2}\right| \leq\|Q\| \psi_{\ell}(\gamma+\mu)+\varepsilon \Psi_{\ell} .
$$

Based on (52) and (54), we can obtain the following assertion:
$\left\|S_{1} x_{1}+S_{2} x_{2}\right\|_{y} \leq|Q| \max \left\{\psi_{0}(\gamma+\mu), \psi_{\ell}(\gamma+\mu)\right\}+\varepsilon \max \left\{\Psi_{0}, \Psi_{\ell}\right\}$.

That is,

$$
\left\|S_{1} x_{1}+S_{2} x_{2}\right\|_{\mathscr{Y}} \leq \varepsilon, \quad \forall x_{1}, x_{2} \in \mathscr{C}_{\varepsilon}, \forall t \in[1, T] .
$$

Hence, the first condition given in Theorem 1 is satisfied. For the purpose of showing the second condition of the same theorem which requires to prove that $S_{2}$ is a contraction mapping, we intend to take into account the term $\mid S_{2} x_{1}-$ $S_{2} x_{2}$ I in accordance with the following manner:

$$
\begin{aligned}
\left|S_{2} x_{1}-S_{2} x_{2}\right| \leq & { }^{H} J^{\gamma}\left(\frac{|\beta(t)|\left|x_{1}(t)-x_{2}(t)\right|}{|\alpha(t)|}\right)+\frac{|\chi(t, T)|}{|\chi(\zeta, T)|}\left(\left|a_{1}\right|^{H} J^{\rho_{1}}\left|x_{1}(\zeta)-x_{2}(\zeta)\right|+{ }^{H} J^{\gamma}\left(\frac{|\beta(\zeta)|\left|x_{1}(\zeta)-x_{2}(\zeta)\right|}{|\alpha(\zeta)|}\right)\right) \\
& +\frac{|\chi(t, \zeta)|}{|\chi(T, \zeta)|}\left(\left|a_{2}\right|^{H} J^{\rho_{2}}\left|x_{1}(T)-x_{2}(T)\right|+{ }^{H} J^{\gamma}\left(\frac{|\beta(T)|\left|x_{1}(T)-x_{2}(T)\right|}{|\alpha(T)|}\right)\right) \\
\leq & {\left[\frac{\beta^{*} \log ^{\gamma} T}{\alpha_{1} \Gamma(\gamma+1)}+\frac{\chi_{0}(T)}{|\chi(\zeta, T)|}\left(\frac{\left|a_{1}\right| \log ^{\rho_{1}} \zeta}{\Gamma\left(\rho_{1}+1\right)}+\frac{\beta^{*} \log ^{\gamma} \zeta}{\alpha_{1} \Gamma(\gamma+1)}\right)+\frac{\chi_{0}(\zeta)}{|\chi(\zeta, T)|}\left(\frac{\left|a_{2}\right| \log ^{\rho_{2}} T}{\Gamma\left(\rho_{2}+1\right)}+\frac{\beta^{*} \log ^{\gamma} T}{\alpha_{1} \Gamma(\gamma+1)}\right)\right]\left|x_{1}-x_{2}\right| . }
\end{aligned}
$$

In other words,

$$
\left|S_{2} x_{1}-S_{2} x_{2}\right| \leq \Psi_{0}\left|x_{1}-x_{2}\right| .
$$

Similarly, we can attain the following inequality:

$$
\left|{ }^{\mathrm{CH}} D^{\ell} S_{2} x_{1}-{ }^{\mathrm{CH}} D^{\ell} S_{2} x_{2}\right| \leq \Psi_{\ell}\left|x_{1}-x_{2}\right| .
$$

Again, applying (58) and (59) into the norm $\|\cdot\|_{y}$ defined above yields

$$
\left\|S_{1} x_{1}-S_{2} x_{2}\right\|_{y}=\max \left\{\Psi_{0}, \Psi_{\ell}\right\}\left\|x_{1}-x_{2}\right\|_{y} .
$$

Due to $\max \left\{\Psi_{0}, \Psi_{\ell}\right\}<1$ as we previously assumed, then $\mathrm{S}_{2}$ is indeed contraction mapping.

In the meantime, to address the last condition of Theorem 1, we should first note that $S_{1}$ is a continuous operator on $\mathscr{C}_{\varepsilon}$ due to that the function $h$ so does. Therefore, the rest claim that should be proved is to show that such operator is compact. In accordance with Lemma 5 , it is sufficient to prove that $S_{1} \mathscr{C}_{\varepsilon}$ is equicontinuous and uniformly bounded. In regard to the boundedness issue, its enough to track the following assertions:

$$
\begin{aligned}
\left\|S_{1} x\right\|_{y} & =\max \left\{\max _{1 \leq t \leq T}\left|S_{1} x(t)\right|, \max _{1 \leq t \leq T}\left|{ }^{\mathrm{CH}} D^{\ell} S_{1} x(t)\right|\right\} \\
& \leq\|Q\| \max \left\{\psi_{0}(\gamma+\mu), \psi_{\ell}(\gamma+\mu)\right\} .
\end{aligned}
$$

Thus, it remains to prove that $S_{1}$ is an equicontinuous operator. For this purpose, we intend to estimate the term $\left|S_{1} x\left(t_{2}\right)-S_{1} x\left(t_{1}\right)\right|$, where $t_{1}, t_{2} \in[1, T]$ with $t_{2}>t_{1}$, as follows: 


$$
\begin{aligned}
\left|S_{1} x\left(t_{2}\right)-S_{1} x\left(t_{1}\right)\right| \leq & \frac{1}{\Gamma(\gamma+\mu)} \int_{1}^{t_{1}}\left[\left(\log \frac{t_{2}}{s}\right)^{\gamma+\mu-1}-\left(\log \frac{t_{1}}{s}\right)^{\gamma+\mu-1}\right] \frac{\left|h\left(s, x_{1}(s),{ }^{\mathrm{CH}} D^{\ell} x_{1}(s)\right)\right|}{|\alpha(s)|} \frac{\mathrm{d} s}{s} \\
& +\frac{1}{\Gamma(\gamma+\mu)} \int_{t_{1}}^{t_{2}}\left(\log \frac{t_{2}}{s}\right)^{\gamma+\mu-1} \frac{\left|h\left(s, x(s),{ }^{\mathrm{CH}} D^{\ell} x(s)\right)\right|}{|\alpha(s)|} \frac{\mathrm{d} s}{s} \\
& +\frac{\left|\chi\left(t_{2}, T\right)-\chi\left(t_{1}, T\right)\right|}{|\chi(\zeta, T)| \Gamma(\gamma+\mu)} \int_{1}^{\zeta}\left(\log \frac{\zeta}{s}\right)^{\gamma+\mu-1} \frac{\left|h\left(s, x(s),{ }^{\mathrm{CH}} D^{\ell} x(s)\right)\right|}{|\alpha(s)|} \frac{\mathrm{d} s}{s} \\
& +\frac{\left|\chi\left(t_{2}, \zeta\right)-\chi\left(t_{1}, \zeta\right)\right|}{|\chi(T, \zeta)| \Gamma(\gamma+\mu)} \int_{1}^{T}\left(\log \frac{T}{s}\right)^{\gamma+\mu-1} \frac{\left|h\left(s, x(s),{ }^{\mathrm{CH}} D^{\ell} x(s)\right)\right|}{|\alpha(s)|} \frac{\mathrm{d} s}{s} \\
\leq & \frac{|Q|}{\alpha_{1} \Gamma(\gamma+\mu+1)}\left(\log { }^{\gamma+\mu} t_{2}-\log { }^{\gamma+\mu} t_{1}\right)+\frac{|Q|}{\alpha_{1} \Gamma(\gamma+\mu+1)}\left(\log \frac{t_{2}}{t_{1}}\right)^{\gamma+\mu} \\
& +\frac{\left|\chi\left(t_{2}, T\right)-\chi\left(t_{1}, T\right)\right||| \mathrm{Q} \||\log \zeta|^{\gamma+\mu}}{\alpha_{1}|\chi(\zeta, T)| \Gamma(\gamma+\mu+1)}+\frac{\left|\chi\left(t_{2}, \zeta\right)-\chi\left(t_{1}, \zeta\right)\right||\mathrm{Q}||\log T|^{\gamma+\mu}}{\alpha_{1}|\chi(T, \zeta)| \Gamma(\gamma+\mu+1)=0}
\end{aligned}
$$

Note that with aiding the results in Lemma 9, the righthand side goes uniformly to zero as $t_{2} \longrightarrow t_{1}$. By the same method, we can deduce that

$$
\left|{ }^{\mathrm{CH}} D^{\ell} S_{1} x\left(t_{2}\right)-{ }^{\mathrm{CH}} D^{\ell} S_{1} x\left(t_{1}\right)\right|,
$$

goes uniformly to zero as $t_{2} \longrightarrow t_{1}$. Thus, based on Lemma 5 , one can conclude that $S_{1}$ is an equicontinuous operator, proving the third condition of Krasnoselskii's Theorem 1, and hence there is at least one solution of the main problems (1) and (2) on $[1, T]$, as required.

4.2. Noncompactness Case. The existence of solution for the FoLE in the case of noncompactness of solution can be further explored by utilizing Kuratowski's measure of noncompactness through applying Darbo's fixed point Theorem 2. This matter can be addressed by considering the next existence result.

Theorem 4. Assume that the two assumptions $\mathbf{H}_{1}$ and $\mathbf{H}_{3}$ are satisfied. If

$$
\max \left\{L \psi_{0}(\gamma+\mu)+\Psi_{0}, L \psi_{\ell}(\gamma+\mu)+\Psi_{\ell}\right\}<1,
$$

where $\psi_{\rho}(\cdot)$ and $\Psi_{\rho}$ are defined in (44) and (45), respectively, then the main problems (1) and (2) have at least one solution on $[1, T]$.

Proof. Due to the matter of finding a solution of the main problem is just considered as a fixed point problem, we define operator $S: \mathscr{B} \longrightarrow \mathscr{B}$ and its fractional derivative $D^{\ell} S: \mathscr{B} \longrightarrow \mathscr{B}$ as previously mentioned in (31) and (31), respectively. To begin the proof of this result, we first need to show that the operator $S: \mathscr{C}_{\varepsilon} \longrightarrow \mathscr{C}_{\varepsilon}$ is continuous where $\mathscr{C}_{\varepsilon}$ is defined in (47), but with radius

$$
\varepsilon \geq \frac{N \max \left\{\psi_{0}(\gamma+\mu), \psi_{\ell}(\gamma+\mu)\right\}}{-\max \left\{L \psi_{0}(\gamma+\mu)+\Psi_{0}, L \psi_{\ell}(\gamma+\mu)+\Psi_{\ell}\right\}},
$$

and $N=\sup _{t \in[1, T]}|h(t, 0,0)|<\infty$. Obviously, the set $\mathscr{C}_{\varepsilon}$ is closed, bounded, and convex nonempty subset of the Banach space $\mathscr{Y}$. Now, we need to prove that $\mathrm{S}\left(\mathscr{C}_{\varepsilon}\right) \subset \mathscr{C}_{\varepsilon}$ and $S$ is continuous on $\mathscr{C}_{\varepsilon}$.

To show that $S: \mathscr{C}_{\varepsilon} \longrightarrow \mathscr{C}_{\varepsilon}$ is continuous, we let $\left\{x_{n}\right\}_{n \in \mathbb{N}}$ to be a sequence of a Banach space $y$ such that $x_{n} \longrightarrow x$ as $n \longrightarrow \infty$. If $t \in[1, T]$, then

$$
\begin{aligned}
\left|\left(S_{1} x_{n}\right)(t)-\left(S_{1} x\right)(t)\right| \leq & \frac{1}{\alpha_{1}} J^{\gamma+\mu}\left|h\left(t, x_{n}(t),{ }^{\mathrm{CH}} D^{\ell} x_{n}(t)\right)-h\left(t, x(t),{ }^{\mathrm{CH}} D^{\ell} x(t)\right)\right| \\
& +\frac{1}{\alpha_{1}} J^{\gamma+\mu}\left|h\left(\zeta, x_{n}(\zeta),{ }^{\mathrm{CH}} D^{\ell} x_{n}(\zeta)\right)-h\left(\zeta, x(\zeta),{ }^{\mathrm{CH}} D^{\ell} x(\zeta)\right)\right| \\
& +\frac{1}{\alpha_{1}} J^{\gamma+\mu}\left|h\left(T, x_{n}(T),{ }^{\mathrm{CH}} D^{\ell} x_{n}(T)\right)-h\left(T, x(t),{ }^{\mathrm{CH}} D^{\ell} x(T)\right)\right| .
\end{aligned}
$$


Consequently, from the assumption $\mathbf{H}_{3}$, we have

$$
\begin{aligned}
\left|\left(S_{1} x_{n}\right)(t)-\left(S_{1} x\right)(t)\right| & \leq \frac{L\left\|x_{n}-x\right\|_{y}}{\alpha_{1} \Gamma(\gamma+\mu+1)}\left(\log ^{\gamma+\mu} T+\frac{\chi(T) \log ^{\gamma+\mu} \zeta+\chi(\zeta) \log ^{\gamma+\mu} T}{|\chi(\zeta, T)|}\right) \\
& \leq L \psi_{0}(\gamma+\mu)\left\|x_{n}-x\right\|_{y} .
\end{aligned}
$$

Also,

$$
\begin{aligned}
\left|\left(S_{2} x_{n}\right)(t)-\left(S_{2} x\right)(t)\right| \leq & \frac{\beta^{*}{ }^{\prime}}{\alpha_{1}} J^{\gamma}\left|x_{n}(t)-x(t)\right|+\frac{|\chi(t, T)|}{|\chi(T, \zeta)|}\left\{\left|a_{1}\right|^{H} J^{\rho_{1}}\left|x_{n}(\zeta)-x(\zeta)\right|+\frac{\beta^{*}{ }^{\prime}}{\alpha_{1}} J^{\gamma}\left|x_{n}(\zeta)-x(\zeta)\right|\right\} \\
& +\frac{|\chi(t, \zeta)|}{|\chi(T, \zeta)|}\left\{\left|a_{2}\right|^{H} J^{\rho_{2}}\left|x_{n}(T)-x(T)\right|+\frac{\beta^{*}{ }^{H}}{\alpha_{1}} J^{\gamma}\left|x_{n}(T)-x(T)\right|\right\} \\
\leq & \frac{\beta^{*} \log ^{\gamma} T}{\alpha_{1} \Gamma(\gamma+1)}\left\|x_{n}-x\right\|_{y}+\frac{\chi(T)}{|\chi(\zeta, T)|}\left[\frac{\left|a_{1}\right| \log ^{\rho_{1}} \zeta}{\Gamma\left(\rho_{1}+1\right)}+\frac{\beta^{*} \log ^{\gamma} \zeta}{\alpha_{1} \Gamma(\gamma+1)}\right]\left\|x_{n}-x\right\|_{y} \\
& +\frac{\chi(\zeta)}{|\chi(\zeta, T)|}\left[\frac{\left|a_{2}\right| \log ^{\rho_{2}} T}{\Gamma\left(\rho_{2}+1\right)}+\frac{\beta^{*} \log ^{\gamma} T}{\alpha_{1} \Gamma(\gamma+1)}\right]\left\|x_{n}-x\right\|_{y}=\Psi_{0}\left\|x_{n}-x\right\|_{y} .
\end{aligned}
$$

Hence, we deduce that

$$
\left\|S_{1} x_{n}-S_{1} x\right\| \leq\left[L \psi_{0}(\gamma+\mu)+\Psi_{0}\right]\left|x_{n}-x\right|_{y} .
$$

Similarly, we can attain the following assertion:

$\left\|{ }^{\mathrm{CH}} D^{\ell} x_{n}-{ }^{\mathrm{CH}} D^{\ell} x\right\| \leq\left[L \psi_{\ell}(\gamma+\mu)+\Psi_{\ell}\right]\left\|x_{n}-x\right\|_{y}$.

In conclusion, we get

$$
\begin{gathered}
\left\|S x_{n}-S x\right\|_{y} \leq \max \left\{L \psi_{0}(\gamma+\mu)+\Psi_{0}, L \psi_{\ell}(\gamma+\mu)\right. \\
\left.+\Psi_{\ell}\right\}\left\|x_{n}-x\right\|_{y} .
\end{gathered}
$$

As $n \longrightarrow \infty$, then $\left\|S x_{n}-S x\right\|_{y} \longrightarrow 0$ which implies that the operator $S$ is continuous on the space $\mathcal{Y}$.

In order to prove that $\mathrm{S}\left(\mathscr{C}_{\varepsilon}\right) \subset \mathscr{C}_{\varepsilon}$, take $x \in \mathscr{C}_{\varepsilon}$. In view of (61), we get

$$
\left\|S_{1} x\right\|_{y} \leq(L \varepsilon+N) \max \left\{\psi_{0}(\gamma+\mu), \psi_{\ell}(\gamma+\mu)\right\} .
$$

Indeed, for $t \in[1, T]$, we have

$$
\begin{aligned}
\left|S_{2} x(t)\right| \leq & \frac{\beta^{*} \varepsilon \log ^{\gamma+\mu} T}{\alpha_{1} \Gamma(\gamma+1)}+\frac{\chi(T)}{|\chi(\zeta, T)|}\left\{\frac{\left|a_{1}\right| \varepsilon \log ^{\rho_{1}} \zeta}{\Gamma\left(\rho_{1}+1\right)}+\frac{\beta^{*} \varepsilon \log ^{\gamma} \zeta}{\alpha_{1} \Gamma(\gamma+1)}\right\} \\
& +\frac{\chi(\zeta)}{|\chi(\zeta, T)|}\left\{\frac{\left|a_{2}\right| \varepsilon \log ^{\rho_{2}} T}{\Gamma\left(\rho_{2}+1\right)}+\frac{\beta^{*} \varepsilon \log ^{\gamma} T}{\alpha_{1} \Gamma(\gamma+1)}\right\} \varepsilon \Psi_{0} .
\end{aligned}
$$

Similarly, we can deduce that $\left|{ }^{\mathrm{CH}} D^{\ell} S_{2} x(t)\right| \leq \varepsilon \Psi_{\ell}$ which with the inequality above lead to

$$
\left\|S_{2} x\right\|_{y} \leq \varepsilon \max \left\{\Psi_{0}, \Psi_{\ell}\right\} \text {. }
$$

Based on (72) and (74), we observe that $\|S x\|_{y} \leq \varepsilon$ which concludes that $\mathrm{S}\left(\mathscr{C}_{\varepsilon}\right) \subset \mathscr{C}_{\varepsilon}$.

The final step of this proof is to show that the operator $S: \mathscr{C}_{\varepsilon} \longrightarrow \mathscr{C}_{\varepsilon}$ satisfies the inequality of the Kuratowski 
measure of noncompactness in Theorem 2. To do this, let $W$ be a closed subset of $\mathscr{C}_{\varepsilon}$ such that there exists $W_{i}, i=1,2, \ldots, n$ and $W \subseteq \cup_{i=1}^{n} W_{i}$. Then,

$$
\begin{aligned}
\sigma_{y}(\mathrm{SW})= & \inf \left\{\kappa: \sup \left\{\left\|S x_{1}(t)-S x_{2}(t)\right\|_{y} \leq \kappa, x_{1}, x_{2} \in W_{i}\right\}\right\} \\
= & \inf \left\{\kappa: \sup \left\{\max \left\{\left\|S x_{1}(t)-S x_{2}(t)\right\|,\left\|{ }^{\mathrm{CH}} D^{\ell} S x_{1}(t)-{ }^{\mathrm{CH}} D^{\ell} S x_{2}(t)\right\|\right\}, x_{1}, x_{2} \in W_{i}\right\} \leq \kappa\right\} \\
= & \max \left\{\inf \left\{\kappa: \sup \left\{\left\|S x_{1}(t)-S x_{2}(t)\right\|, x_{1}, x_{2} \in W_{i}\right\}\right\} \leq \kappa\right\} \\
& \left.\inf \left\{\kappa: \sup \left\{\left\|{ }^{\mathrm{CH}} D^{\ell} S x_{1}(t)-{ }^{\mathrm{CH}} D^{\ell} S x_{2}(t)\right\|, x_{1}, x_{2} \in W_{i}\right\} \leq \kappa\right\}\right\} \\
= & \left.\left.\max \{\sigma(\mathrm{SW})), \sigma\left({ }^{\mathrm{CH}} D^{\ell} \mathrm{SW}\right)\right)\right\} .
\end{aligned}
$$

Also, let there exist $U \subseteq \cup_{i=1}^{n} U_{i}$ and $V \subseteq \cup_{i=1}^{n} V_{i}$ such that

$$
\begin{aligned}
& U_{i}=\left\{\mathrm{H} J^{\mu}\left[\frac{h\left(t, x_{i}(t),{ }^{\mathrm{CH}} D^{\ell} x_{i}(t)\right)}{\alpha(t)}\right]: x_{i} \in W_{i}, t \in[1, T]\right\}, \\
& V_{i}=\left\{h\left(t, x_{i}(t),{ }^{\mathrm{CH}} D^{\ell} x_{i}(t)\right): x_{i} \in W_{i}, t \in[1, T]\right\} .
\end{aligned}
$$

In view of continuity of the functions $h$ and $\alpha$, the sets $U$ and $V / \alpha$ are bounded and equicontinuous and so with the last item of Lemma 4, we obtain

$$
\sigma(U) \leq{ }^{H} J^{\mu} \sigma\left(\frac{V}{\alpha(t)}\right) \leq \frac{1}{\alpha_{1}} J^{\mu} \sigma(V),
$$

and with Definition 3, we get

$$
\begin{aligned}
\sigma(V) & =\inf \left\{\kappa: \sup \left\{\left\|h\left(t, x_{i 1}(t),{ }^{\mathrm{CH}} D^{\ell} x_{i 1}(t)\right)-h\left(t, x_{i 2}(t),{ }^{\mathrm{CH}} D^{\ell} x_{i 2}(t)\right)\right\| \leq \kappa, x_{i 1}, x_{i 2} \in W_{i}\right\}\right\} \\
& \leq \inf \left\{\kappa: \sup \left\{L \max \left\{\left\|x_{i 1}-x_{i 2}\right\|,\left\|{ }^{\mathrm{CH}} D^{\ell} x_{i 1}-{ }^{\mathrm{CH}} D^{\ell} x_{i 2}\right\|\right\} \leq \kappa, x_{i 1}, x_{i 2} \in W_{i}\right\}\right\} \\
& =\operatorname{Linf}\left\{\kappa / L: \sup \left\{\left|x_{i 1}-x_{i 2}\right| y \leq \frac{\kappa}{L}, x_{i 1}, x_{i 2} \in W_{i}\right\}\right\} \\
& =L \sigma(W),
\end{aligned}
$$

which implies that

From the second and third items of Lemma 4, we get

$$
\sigma(U) \leq \frac{L \log ^{\mu} t}{\alpha_{1} \Gamma(\mu+1)} \sigma(W) .
$$

$$
\begin{aligned}
\sigma\left(S_{1} W\right) \leq & \sigma\left({ }^{H} J^{\gamma}\left[\frac{{ }^{H} J^{\mu} h\left(t, x(t),{ }^{\mathrm{CH}} D^{\ell} x(t)\right)}{\alpha(t)}\right]\right)+\frac{\chi(T)}{|\chi(\zeta, T)|} \sigma\left({ }^{H} J^{\gamma}\left[\frac{{ }^{H} J^{\mu} h\left(\zeta, x(\zeta),{ }^{\mathrm{CH}} D^{\ell} x(\zeta)\right)}{\alpha(t)}\right]\right) \\
& +\frac{\chi(\zeta)}{|\chi(\zeta, T)|} \sigma\left({ }^{H} J^{\gamma}\left[\frac{{ }^{H} J^{\mu} h\left(T, x(T),{ }^{\mathrm{CH}} D^{\ell} x(T)\right)}{\alpha(t)}\right]\right), \quad x \in W \\
\leq & { }^{H} J^{\gamma} \sigma(U)(t)+{ }^{H} J^{\gamma} \gamma \sigma(U)(T)+{ }^{H} J^{\gamma} \sigma(U)(\zeta) \\
\leq & \frac{L}{\alpha_{1} \Gamma(\gamma+\mu+1)}\left(\log ^{\gamma+\mu} T+\frac{\chi(T) \log ^{\gamma+\mu} \zeta+\chi(\text { zeta }) \log ^{\gamma+\mu} T}{|\chi(\zeta, T)|}\right) \sigma(W) \\
= & L \psi_{0}(\gamma+\mu) \sigma(W) .
\end{aligned}
$$


In the similar way, we have

$$
\begin{aligned}
\sigma\left(S_{2} W\right) \leq & \sigma\left[{ }^{H} J^{\gamma}\left(\frac{\beta(t) x(t)}{\alpha(t)}\right)+\frac{\chi(t, T)}{\chi(T, \zeta)}\left\{a_{1}{ }^{H} J^{\rho_{1}} x(\zeta)+{ }^{H} J^{\gamma}\left(\frac{\beta(\zeta) x(\zeta)}{\alpha(\zeta)}\right)\right\}\right. \\
& \left.+\frac{\chi(t, \zeta)}{\chi(\zeta, T)}\left\{a_{2}{ }^{H} J^{\rho_{2}} x(T)+{ }^{H} J^{\gamma}\left(\frac{\beta(T) x(T)}{\alpha(T)}\right)\right\}, x \in W\right] \\
\leq & \Psi_{0} \sigma(W) .
\end{aligned}
$$

Hence,

$\sigma(\mathrm{SW}) \leq \sigma\left(S_{1} W\right)+\sigma\left(S_{2} W\right) \leq\left(L \psi_{0}(\gamma+\mu)+\Psi_{0}\right) \sigma(W)$.

Similarly, we can follow the same procedure in regard to ${ }^{\mathrm{CH}} D^{\ell} \mathrm{SW}$. In other word, we can obtain the following assertion:

$$
\begin{aligned}
& \sigma\left({ }^{{ }^{\mathrm{CH}}} D^{\ell} \mathrm{SW}\right) \leq \sigma\left({ }^{{ }^{\mathrm{CH}}} D^{\ell} \mathrm{S}_{1} W\right)+\sigma\left({ }^{\mathrm{CH}} D^{\ell} \mathrm{S}_{2} W\right) \\
& \leq\left(\psi_{\ell}(\gamma+\mu)+\Psi_{\ell}\right) \sigma(W) .
\end{aligned}
$$

Substituting (82) and (83) into (75) concludes that $\sigma_{\mathscr{y}}(\mathrm{SW}) \leq \max \left\{L \psi_{0}(\gamma+\mu)+\Psi_{0}, \psi_{\ell}(\gamma+\mu)+\Psi_{\ell}\right\} \sigma(W)$.
In view of the Darbo fixed point theorem, we confirm that the operator $S$ has a fixed point which represents a solution of the main problems (1) and (2).

\section{Numerical Examples}

In this part, the main B.V.P. reported in (1) and (2) is addressed via two numerical examples. These examples are handled for demonstrating the two results formulated in the previous section.

5.1. Example 1. Consider the B.V.P. as follows:

$$
{ }^{c} D^{7 / 4}\left(e^{-t c} D^{2 / 3}+\left(\frac{1}{30} t^{1 / 2}+\frac{1}{100}\right)\right) x(t)=\frac{e^{-t}}{\left(5+e^{t}\right)}\left(\frac{\left|x(t)^{c} D^{1 / 4} x(t)\right|}{1+\left|x(t)^{c} D^{1 / 4} x(t)\right|}\right), \quad t \in[1,2],
$$

subject to the conditions:

$$
x(1)=0, x\left(\frac{3}{2}\right)=\frac{7}{1000} J^{2 / 3} x\left(\frac{3}{2}\right), x(2)=\frac{13}{500} J^{2 / 3} x(2) .
$$

Comparing this problem with the main problem yields to outline that $\mu=7 / 4, \gamma=2 / 3, \ell=1 / 4, \zeta=3 / 2, T=2$, $a_{1}=7 / 1000$, and $a_{2}=13 / 500, \rho_{1}=2 / 5, \rho_{2}=4 / 7$. On the other hand, we have $\alpha(t)=t^{3 / 2}$ and $\beta(t)=1 / 30 t^{1 / 2}+1 / 100$, which give $\alpha_{1}=e^{-2}, \alpha_{2}=e^{-1}$ and $\beta^{*}=0.0571$. Moreover, we have

$$
h\left(t, x(t),{ }^{c} D^{1 / 2} x(t)\right)=\frac{e^{-t}}{\left(5+e^{t}\right)}\left(\frac{\left|x(t)^{c} D^{1 / 2} x(t)\right|}{1+\left|x(t)^{c} D^{1 / 2} x(t)\right|}\right),
$$

which is continuous and satisfies

$$
\left|h\left(t, x(t),{ }^{c} D^{1 / 2} x(t)\right)\right| \leq Q(t)=\frac{1}{e^{t}\left(5+e^{t}\right)} .
$$

This implies that the second condition $\mathrm{H}_{2}$ is satisfied with $\|Q\|=0.0476634$. By carrying out Mathematica software, it is easy to obtain $|\chi(T, \zeta)|=2.03966$,

$$
\begin{aligned}
\chi_{0}(T) & =0.602723, \chi_{0}(\zeta)=0.403831, \\
\chi_{\ell}(T) & =1.21289, \chi_{\ell}(\zeta)=0.689803, \\
\psi_{0}(\gamma) & =9.00499, \psi_{\ell}(\gamma)=11.3628, \\
\Psi_{0} & =0.566214, \Psi_{\ell}=0.741573,
\end{aligned}
$$

which implies that $\max \left\{\Psi_{0}, \Psi_{\ell}\right\}=0.741573<1$. Therefore, based on Theorem 1, we conclude that the B.V.P. (85) and (86) have at least one solution on $[1,2]$.

5.2. Example 2. Consider the following B.V.P.:

$$
{ }^{c} D^{5 / 3}\left(e^{-t c} D^{1 / 2}+\left(t^{1 / 2}+1\right)\right) x(t)=\left(\frac{|x(t)|}{|x(t)|+1}\right) \frac{\sin ^{4} t}{\left(10^{t}+9\right)^{2}}+\left(\frac{\left|{ }^{c} D^{1 / 3} x(t)\right|}{\left|{ }^{c} D^{1 / 3} x(t)\right|+1}\right) \frac{\cos ^{2} t}{(t+1)^{2}}+\frac{t^{2}}{5}, \quad t \in[1, \pi],
$$


subject to the conditions:

$$
x(1)=0, x\left(\frac{4}{3}\right)=\frac{9}{100} J^{1 / 2} x\left(\frac{4}{3}\right), x(\pi)=\frac{5}{171} J^{1 / 2} x(\pi) .
$$

Comparing (90) and (91) with (1) and (2) leads us to obtain $\mu=5 / 3, \gamma=1 / 2, \ell=1 / 3, \zeta=4 / 3, T=\pi, a_{1}=9 / 100$, $a_{2}=5 / 171, \rho_{1}=3 / 7$, and $\rho_{2}=3 / 8$. In the meantime, we obtain $\alpha(t)=e^{-t}$ and $\beta(t)=t^{1 / 2}+1$ which implies $\alpha_{1}=e^{-\pi}$, $\alpha_{2}=e^{-1}$, and $\beta^{*}=\sqrt{\pi}+1$. Furthermore, we have

$$
h\left(t, x(t),{ }^{c} D^{1 / 3} x(t)\right)=\left(\frac{|x(t)|}{|x(t)|+1}\right) \frac{\sin ^{4} t}{\left(10^{t}+9\right)^{2}}+\left(\frac{\left|{ }^{c} D^{1 / 3} x(t)\right|}{\left|{ }^{c} D^{1 / 3} x(t)\right|+1}\right) \frac{\cos ^{2} t}{(t+1)^{2}}+\frac{t^{2}}{5},
$$

which is a continuous function. In order to see that it satisfies the third condition $\mathrm{H}_{3}$, we have

$$
\begin{aligned}
& \left|h\left(t, x_{1}(t),{ }^{c} D^{1 / 3} x_{1}(t)\right)-h\left(t, x_{2}(t),{ }^{c} D^{1 / 3} x_{2}(t)\right)\right| \\
\leq & \frac{1}{\left(10^{t}+9\right)}\left|x_{1}(t)-x_{2}(t)\right|+\frac{1}{t(t+1)^{2}}\left|{ }^{c} D^{1 / 3} x_{1}(t)-{ }^{c} D^{1 / 3} x_{2}(t)\right| \\
\leq & \frac{1}{19^{2}}\left|x_{1}(t)-x_{2}(t)\right|+\frac{1}{2^{2}}\left|{ }^{c} D^{1 / 3} x_{1}(t)-{ }^{c} D^{1 / 3} x_{2}(t)\right| \\
\leq & \left(\frac{1}{19^{2}}+\frac{1}{2^{2}}\right) \max \left\{\left|x_{1}(t)-x_{2}(t)\right|,\left|{ }^{c} D^{1 / 3} x_{1}(t)-{ }^{c} D^{1 / 3} x_{2}(t)\right|\right\},
\end{aligned}
$$

which implies that the function $h$ satisfies the third condition $H_{3}$ with $L=365 / 1444$. By carrying out Mathematica software, it is easy to obtain $|\chi(T, \zeta)|=22.0176$,

$$
\begin{aligned}
\chi_{0}(T) & =1.51194, \chi_{0}(\zeta)=0.694439, \\
\chi_{\ell}(T) & =6.60417, \chi_{\ell}(\zeta)=1.58684, \\
\psi_{0}(\gamma+\mu) & =29.7801, \psi_{\ell}(\gamma+\mu)=32.902, \\
\Psi_{0} & =0.181856, \Psi_{\ell}=0.582983,
\end{aligned}
$$

which implies that

$$
\max \left\{L \psi_{0}(\gamma+\mu)+\Psi_{0}, L \psi_{\ell}(\gamma+\mu)+\Psi_{\ell}\right\}=\max \{0.510466,0.946042\}=0.946042<1
$$

Hence, according to Theorem 4, we deduce that there is at least one solution to the B.V.P. (90) and (91) on the interval $[1, \pi]$.

\section{Conclusion}

This paper has introduced two novel results associated with the existence of solutions for the fractional-order Langevin problem that has been formulated in the sense of Caputo-Hadamard fractional-order derivative operator. These two results have been shown in light of two well-known fixed point theorems, namely, the Krasnoselskii's theorem in the case of compactness and Darbo's theorem in the case of noncompactness. Two numerical examples have been demonstrated for completeness and illustrate the implementations of our idea on scientific problems. Here, we applied this idea on fractional differential equations; we think that applying this idea on the fractional differential inclusion will be useful in the future study.

\section{Data Availability}

The qualitative data used to support the findings of this study are included within the article. 


\section{Conflicts of Interest}

The authors declare that they have no conflicts of interest.

\section{Authors' Contributions}

All authors contributed equally to this work. All authors read and approved the final manuscript.

\section{Acknowledgments}

This project was funded by the Deanship of Scientific Research (DSR), King Abdulaziz University, Jeddah, Saudi Arabia, under grant no. KEP-PhD-60-130-42. The authors, therefore, acknowledge with thanks DSR's technical and financial support.

\section{References}

[1] D. Baleanu, S. S. Sajjadi, A. Jajarmi, and J. Asad, "The fractional dynamics of a linear triatomic molecule," Romanian Reports in Physics, vol. 73, no. 1, p. 105, 2021.

[2] G. Wang and T. Wang, "On a nonlinear Hadamard type fractional differential equation with p-Laplacian operator and strip condition," The Journal of Nonlinear Science and Applications, vol. 9, no. 7, pp. 5073-5081, 2016.

[3] P. Borisut, P. Kumam, I. Ahmed, and K. Sitthithakerngkiet, "Nonlinear Caputo fractional derivative with nonlocal riemann-liouville fractional integral condition via fixed point theorems," Symmetry, vol. 11, no. 6, p. 829, 2019.

[4] I. Podlubny, Fractional Differential Equations, Academic Press, New York, NY, USA, 1999.

[5] A. A. Kilbas, H. M. Srivastava, and J. J. Trujillo, Theory and Applications of Fractional Differential Equations, NorthHolland Mathematics Studies; Elsevier, Amsterdam, Netherlands, 2006.

[6] A. Salem and A. Al-dosari, "Existence results of solution for fractional Sturm-Liouville inclusion involving composition with multi-maps," Journal of Taibah University for Science, vol. 14, no. 1, pp. 721-733, 2020.

[7] A. Salem, F. Alzahrani, and A. Al-dosari, "Attainabillity to solve fractional differential inclusion on the half line at resonance," Complexity, vol. 2020, Article ID 9609108, 13 pages, 2020.

[8] A. Babaei, H. Jafari, and A. Liya, "A. Mathematical models of HIV/AIDS and drug addiction in prisons," The European Physical Journal Plus, vol. 135, no. 5, p. 395, 2020.

[9] W. T. Coffey, Y. P. Kalmykov, and J. T. Waldron, The Langevin Equation, World Scientific, Singapore, 2004.

[10] C. Kiataramkul, S. K. Ntouyas, J. Tariboon, and A. Kijjathanakorn, "Generalized Sturm-Liouville and Langevin equations via Hadamard fractional derivatives with anti-periodic boundary conditions," Boundary Value Problems, vol. 2016, no. 1, Article ID 217, 2016.

[11] B. Datsko and V. Gafiychuk, "Complex nonlinear dynamics in subdiffusive activator-inhibitor systems," Communications in Nonlinear Science and Numerical Simulation, vol. 17, no. 4, pp. 1673-1680, 2012.

[12] B. Datsko and V. Gafiychuk, "Complex spatio-temporal solutions in fractional reaction-diffusion systems near a bifurcation point," Fractional Calculus and Applied Analysis, vol. 21, no. 1, pp. 237-253, 2018.
[13] K. G. Wang and M. Tokuyama, "Nonequilibrium statistical description of anomalous diffusion," Physica A: Statistical Mechanics and Its Applications, vol. 265, no. 3-4, pp. 341-351, 1999.

[14] A. Salem and B. Alghamdi, "Multi-Strip and multi-point boundary conditions for fractional Langevin equation," Fractal and Fractional, vol. 4, no. 2, p. 18, 2020.

[15] H. Baghani, "An analytical improvement of a study of nonlinear Langevin equation involving two fractional orders in different intervals," Journal of Fixed Point Theory and Applications, vol. 21, no. 95, pp. 1-11, 2019.

[16] B. J. West and S. Picozzi, "Fractional Langevin model of memory in financial time series," Physical review. E, Statistical, nonlinear, and soft matter physics, vol. 65, Article ID 037106, 2002.

[17] F. Mainradi and P. Pironi, "The fractional Langevin equation: brownian motion revisted," Extracta Mathematicae, vol. 10, pp. 140-154, 1996.

[18] T. Chen, W. Liu, and Z. Hu, "A boundary value problem for fractional differential equation with P-Laplacian operator at resonance," Nonlinear Analysis: Theory, Methods \& Applications, vol. 75, no. 6, pp. 3210-3217, 2012.

[19] A. Salem, "Existence results of solutions for anti-periodic fractional Langevin equation," Journal of Applied Analysis \& Computation, vol. 10, no. 6, pp. 2557-2574, 2020.

[20] S. Liang and J. Zhang, "Positive solutions for boundary value problems of nonlinear fractional differential equations," Nonlinear Analysis: Theory, Methods \& Applications, vol. 71, no. 11, pp. 5545-5550, 2009.

[21] Z. Han, H. Lu, and C. Zhang, "Positive solutions for eigenvalue problems of fractional differential equations with generalized P-Laplacian," Applied Mathematics and Computation, vol. 257, pp. 526-536, 2014.

[22] X. Zhang, L. Wang, and Q. Sun, "Existence of positive solutions for a class of nonlinear fractional differential equations with integral boundary conditions and a parameter," Applied Mathematics and Computation, vol. 226, pp. 708-718, 2014.

[23] B. Ahmad, M. Alghanmi, A. Alsaedi, H. M. Srivastava, and S. N. Ntouyas, "The Langevin equation in terms of generalized Liouville-Caputo derivatives with nonlocal boundary conditions involving a generalized fractional integral," Mathematics, vol. 7, pp. 1-10, 2019.

[24] A. Salem, F. Alzahrani, and M. Alnegga, "Coupled system of nonlinear fractional Langevin equations with multi-point and nonlocal integral boundary conditions," Mathematical Problems in Engineering, vol. 2020, Article ID 7345658, 2020.

[25] A. Salem and B. Alghamdi, "Multi-point and anti-periodic conditions for generalized Langevin equation with two fractional orders," Fractal and Fractional, vol. 3, no. 4, pp. 1-14, 2019.

[26] A. Salem and N. Mshary, "On the existence and uniqueness of solution to fractional-order Langevin equation," Advances in Mathematical Physics, vol. 2020, Article ID 8890575, 2020.

[27] T. Muensawat, S. K. Ntouyas, and J. Tariboon, "Systems of generalized Sturm-Liouville and Langevin fractional differential equations," Advances in Difference Equations, vol. 2017, no. 1, Article ID 63, 2017.

[28] M. Rivero, J. Trujillo, and M. Velasco, "A fractional approach to the Sturm-Liouville problem,” Open Physics, vol. 11, no. 10, pp. 1246-1254, 2013.

[29] S. Aljoudi, B. Ahmad, J. J. Nieto, and A. Alsaedi, "A coupled system of Hadamard type sequential fractional differential equations with coupled strip conditions," Chaos, Solitons \& Fractals, vol. 91, pp. 39-46, 2016. 
[30] F. Jarad, T. Abdeljawad, and D. Baleanu, "Caputo-type modification of the Hadamard fractional derivatives," Advances in Difference Equations, vol. 2012, no. 1, Article ID 142, 2012.

[31] C. Derbazi, H. Hammouche, and H. Hammouche, "CaputoHadamard fractional differential equations with nonlocal fractional integro-differential boundary conditions via topological degree theory," AIMS Mathematics, vol. 5, no. 3, pp. 2694-2709, 2020.

[32] A. Salem and M. Alnegga, "Measure of noncompactness for hybrid Langevin fractional differential equations," Axioms, vol. 9, no. 2, p. 59, 2020.

[33] M. Mursaleen, B. Bilalov, and S. Rizvi, "Applications of measures of noncompactness to infinite system of fractional differential equations," Filomat, vol. 31, no. 11, pp. 3421-3432, 2017.

[34] A. Seemab and M. Ur. Rehman, "Existence of solution of an infinite system of generalized fractional differential equations by Darbo's fixed point theorem," Journal of Computational and Applied Mathematics, vol. 364, Article ID 112355, 2020. 